\title{
The Digital Image Processing Technology in Application of License Plate Recognition Intelligent System
}

\author{
Huangfei Chen, Zhuangying Fang \\ Guangzhou Huali Science and Technology Vocational College \\ Guangzhou of Guangdong Province, China \\ 531028136@qq.com,214126393@qq.com
}

\begin{abstract}
With the development of science and technology and the development of transportation industry, the intelligent traffic management system is becoming more and more updated. A vehicle license plate recognition system has been widely used in people's lives. In this paper, four steps of license plate recognition and their corresponding algorithms are analyzed and studied: the license plate image preprocessing part includes image de-fogging pretreatment, spatial smoothing filtering, image sharpening and other processes; the license plate image positioning methods are compared, and the Radon transform used to correct the license plate image; license plate character segmentation. The character contour method is used to segment the characters and the template matching method is used to license plate character recognition. The improvement of image processing is also proposed. It is proved that the current vehicle recognition system can meet the requirements of accurate license plate recognition, and the robustness and accuracy are improved compared with the general model.
\end{abstract}

Keywords - digital image processing technology ; recognition; Edge detection ; character recognition

\section{INTRODUCTION}

With the improvement of people's living standard, every household now has cars. In order to strengthen the city security prevention and control and the effective management of motor vehicles in the city management, it is necessary to adopt the relevant intelligent traffic management system. Nowadays, in the automobile era, the license plate recognition system has become the inevitable product of human intelligent life. Its application scope is wide: the management of vehicle access in residential areas, the detection of speeding vehicles on highways, parking lot management and so on need the assistance of license plate recognition system.

Due to the limitation of natural lighting, rain and fog, instrument, shooting angle, vehicle speed and other objective conditions, the quality of the recognition image will be greatly affected. Moreover, the shape and color of the license plate are fixed, which brings a certain degree of difficulty to the license plate recognition. So far, there are many different algorithms in the license plate recognition system. How to capture and collect the vehicle images, How to recognize the vehicle license plate quickly, accurately and automatically is a hot topic for many experts and scholars in recent years.

In this paper, the technology of vehicle license plate image recognition is divided into four steps to study: image preprocessing, vehicle license plate location, character segmentation, character recognition ${ }^{[1]}$.

\section{PRE-PROCESSING}

To accurately identify the license plate, we first need to preprocess the license plate image and get the high quality image. Preprocessing is the premise and important part of vehicle license plate image recognition. It is the operation ${ }^{[2]}$ before feature extraction, segmentation and recognition. Generally, the image captured by the camera is in RGB format, which needs some pretreatment such as grayscale, binary, tilt correction, filtering and so on. If fog and haze weather, first image de fog pretreatment.

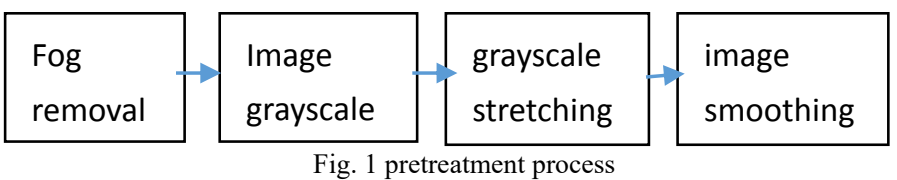

\section{A. image de fog pretreatment}

when collecting pictures in haze weather. The image will become blurred haze ${ }^{[3]}$.In order to identify the image accurately,it must use the haze detection algorithm to detect the image first: Acquisition of image frequency domain information, it used classifier ${ }^{[4]}$ base on the principal compo nent analysis (PCA) and Fisher linear dis criminant analysis (LDA) to detect whether there is fog and haze in the image?. The image is filtered and the power spectrum of the image is analyzed. The spectrum of the image with haze is concentrated near the origin, that is, the low frequency component is more, and the normal weather picture is divergent. For images with haze, a dark priori ${ }^{[5]}$ de-fogging method is used, and a guided filter ${ }^{[6]}$ is used to correct the 
transmittance.The guided filter can clearly retain the edge information of the original image while filtering.

The experimental results show that the time-consuming of defogging detection is only about one-seventh of the defogging processing algorithm. The defogging processing can greatly improve the accuracy of license plate recognition in fog and haze weather without affecting the speed of license plate recognition on the normal picture.

\section{B. grayscale of images}

In order to conduct vertical edge detection, we first use the appropriate Gauss fuzzy radius to denoise the image. Then, grayscale the picture. The existing grayscale function exists in the OpenCV library. The grayscale function is used to grayscale the image, and the pixel is represented by $0 \sim 255$.

(1)EQ ( $f$ ) is a single value incremental function in the range of $0<\mathrm{f}<\mathrm{L}-1$, which ensures that the gray level of the original vehicle license plate image is still in the correct order after enhancement

(2)for $0 \leq \mathrm{f} \leq \mathrm{L}-1$ and $0 \leq \mathrm{g} \leq \mathrm{L}-1$, Condition guarantee that the dynamic range of the gray value of the image before and after transformation is consistent. The cumulative distribution function satisfying the above two conditions can transform the distribution of $F$ from the original vehicle license plate image into the uniform distribution of $\mathrm{G}$. Therefore, the histogram equalization mapping function should be:

$$
\mathrm{G}_{\mathrm{K}}=\mathrm{E}_{\mathrm{Q}}\left(\mathrm{f}_{\mathrm{k}}\right)=\left(\mathrm{n}_{\mathrm{I}} / \mathrm{n}\right)=\mathrm{p}_{\mathrm{f}}\left(\mathrm{f}_{\mathrm{I}}\right),\left(\mathrm{F}, 1,2, \ldots\left(\mathrm{L}_{-1}\right)\right.
$$

According to the gray mapping relation, each pixel of the original image is converted to gray level, and then the histogram equalization of the original image is completed ${ }^{[7]}$.

\section{Binaryzation}

The brightness information of vehicle license plate image after gray processing contains 256 gray levels, and the brightness and shade contrast are distinct. In order to recognize the vehicle license plate, the license plate is completely separated from the whole background, and the image is divided into two parts: the license plate and the background to avoid the interference of background pixels. Therefore, only two gray levels can be used: 0 and 1 . Usually the target pixel value is 1 , and the background pixel value is 0 . After the image is processed in two values, get the image ${ }^{[8]}$ you need. If the pixel value of the gray image before binarization is $\mathrm{f}(\mathrm{x}, \mathrm{y})$, where $(\mathrm{x}, \mathrm{y})$ represents the spatial point coordinates of the gray image before binarization, the pixel value $\mathrm{g}(\mathrm{x}, \mathrm{y})$ of the gray image after binarization is:

$$
\mathrm{g}(\mathrm{x}, \mathrm{y})= \begin{cases}0, & f(x, y)<c \\ 1, & f(x, y) \geq c\end{cases}
$$

Among them, $\mathrm{C}$ is the threshold.

After binarization, we can see that many holes are not connected to the whole graph, and the contrast of gray scale of license plate is obviously enhanced. After binarization, we can see that many holes are not connected to the whole graph, and the contrast of gray scale of license plate is obviously enhanced.

\section{D. filter method}

Filtering is a nonlinear technology of image smoothing. Nowadays, the common way to eliminate random noise without blurring the edges and lines of the image is to smooth the license plate space image, i.e. median filtering method ${ }^{[9]}$. Median filtering, the gray value of each pixel in a sliding window is counted, and then the gray value of the original center is replaced by the median value of the gray value of all pixels in the window. This is a typical and most commonly used non-linear filtering method ${ }^{[10]}$. It can keep the details of the image while eliminating noise and prevent edge blurring. It has more prominent advantages than other linear filtering methods such as neighborhood averaging method, that is, Operations do not require statistical properties of images.

After pretreatment, the license plate image has only two colors, black and white, which greatly enhances the character clarity of the license plate, and makes full preparations for the location and correction of the license plate.

\section{VEHICLE LICENSE PLATE LOCALIZATION AND CORRECTION}

License plate location is to locate the license plate in the image. It is the most important step in the license plate recognition system.

License plate location is the most important step in the license plate recognition system.

At present, the methods of vehicle license plate location are mainly based on edge detection, wavelet analysis, neural network, color feature and texture feature. The most commonly used method is based on edge detection: detect the vertical edges of characters in the license plate. Combined with color localization, the positioning rate can be improved. In order to improve the detection accuracy and extract the outline of the license plate image more accurately, the differential method can be used to sharpen the image of the license plate first, so that the edge information of the outline edge, details and gray level jumping part of the license plate can be enhanced to form a clear object edge.

Edge detection algorithms include Sobel algorithm, Roberts algorithm, Laplacian algorithm, Log algorithm and Canny algorithm. These algorithms are based on the sharp change of the gray level of the vehicle license plate edge for image edge detection.

Roberts edge detection algorithm is a local difference algorithm to find the edge of the algorithm, also known as gradient crossover algorithm, using convolution template as follows:

$$
\mathrm{G}(\mathrm{x}, \mathrm{y})=\left|\left[\begin{array}{rr}
1 & 0 \\
0 & -1
\end{array}\right]\right|+\left|\left[\begin{array}{cc}
0 & 1 \\
-1 & 0
\end{array}\right]\right|
$$

Sobel algorithm is to derive the lateral and longitudinal directional derivatives of the image, and then determine the edge according to the derivative value greater than a certain threshold, and locate the license plate. Based on the previous step, the Sobel algorithm is applied to find out the vertical edge and locate the license plate preliminarily. Sobel finds the vertical edge to locate the license plate, although the effect is 
good, but in front of some cars, there are cross vertical edges, using Sobel can't locate the license plate accurately. In order to solve the license plate location problem of these models, we consider using color to locate. The method of license plate location based on color features can recognize and adjust the segmented area, and finally find the license plate area. In digital image processing, color model is used to describe color, the most commonly used color model is RGB model and HSI model. In order to reduce the interference, RGB model can be used to detect the white area of the picture, HSI model can detect the blue area of the image, and the adaptive adjustment scheme is proposed to identify and adjust the separated area. According to the aspect ratio, the area of blue to white alignment is repeatedly positioned, and finally the license plate area is found.

Combination of Sobel location and color positioning, The accuracy rate increased from $75 \%$ to $95 \%$. Color positioning will use the HSV model ${ }^{[11]}$ and set the threshold to judge. The color positioning process is as follows:

(1) the color space of the captured image is RGB , so it is necessary to transform the image into HSV model. Considering the illumination, the image is preprocessed by histogram equalization.

(2) use 0,1 Identify all pixels of the image, that is, two values. All pixels on the image are traversed. If the $S$ value and $\mathrm{V}$ value are between 0.4 and 1.0 , and the $\mathrm{H}$ value is between 200 and 280, the pixel is marked 0, otherwise it is 1 .

(3) According to the steps and methods of license plate location, the binary image obtained in the second step is closed operation first, and then the outer rectangle of the license plate is intercepted from the contour for further processing.

In traditional Canny algorithm, when calculating the amplitude of gradient, the detected points are directly involved in the arithmetic, and the ability to suppress noise is weak. The improvement should be that the pending detection points do not participate in the arithmetic. The new partial derivative formula is as follows:

$$
P x[i, j]=(I[i, j+1]-I[i, j-1]+I[i+1, j+1]-I[i+1, j-1]) / 2
$$

$$
\text { Py }[i, j]=(I[1, j]-I[i+1, J i]+I[I, j+1]-I[i+1, j+1]) / 2
$$

Although the calculation principle is the same before and after the improvement, the sum is obtained by subtracting the pixel values at both ends of the bidirectional arrow in the same direction, and then the average value is obtained ${ }^{[12]}$. However, the calculation template are different, the amplitude of gradient and direction of gradient are more precise. Secondly, we can consider the "artificial specified double threshold" for the traditional Canny algorithm. According to the content of non maxima resisting image, adaptive double threshold is given. As far as possible, let the high threshold appear outside the non-edge region, and the low threshold appear near the weak edge, effectively repair the high threshold edge.
Because of the angle of image acquisition equipment, a large number of vehicle license plate images will tilt to a certain extent. If not corrected, the image will be distorted when the character recognition stretches, affecting the recognition accuracy. Although the Hough transform can be used, the traditional Hough needs to calculate every pixel and process it for a long time. So the slope $\mathrm{K}$ of both ends of the license plate image is considered to adjust the position of the image and improve the efficiency, that is, the tilt correction method based on Radon transform ${ }^{[13]}$. Radon transform is used to calculate the tilt of license plate and correct it to get high quality license plate image.

The Radon transform along the $\theta$ direction is defined as:

$$
R_{\theta}(X)=\int f(x \cos \theta-y \sin \theta, x \sin \theta+y \cos \theta) d y
$$

According to the characteristics of vehicle license plate image itself: fixed rectangle, deformation degree is small, the original XY plane point mapping to the $\mathrm{AB}$ plane. In this way, a straight line of the original XY plane is located at the same point in the $\mathrm{AB}$ plane. By recording the accumulated thickness of the points in the $\mathrm{AB}$ plane, the existence of the line on the XY plane can be known. In the new plane, the corresponding point accumulation peak can be obtained to obtain the original plane of the salient line set, and then determine the image tilt angle. The transformation of Radon is performed at theta $\theta=-900$ to 900 degrees.

\section{CHARACTER SEGMENTATION}

Character segmentation is to segment all the characters in the license plate, and the character blocks will be recognized.

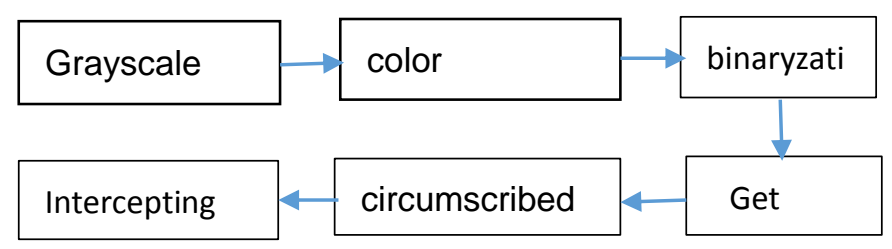

There are many common methods for character segmentation, such as character contour extraction, template matching segmentation, projection, sliding window judgment, connected domain character segmentation and so on.

Fig. 2 character segmentation process

Take the character contour method and its character segmentation is shown in Figure 3.

When extracting English characters, it works very well, but there will be problems in processing Chinese characters. Chinese characters differ from English in structure and structural strokes will break. such as "Su" in Figure 4. English characters can be completely removed, and "Su" is divided into two parts.

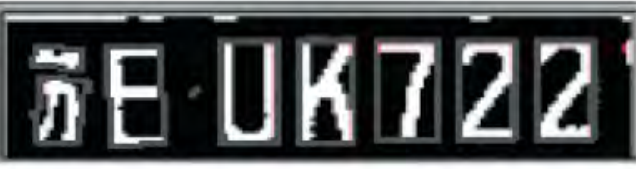

Fig. 3 take contour operation 
Although many Chinese will not appear situation in the above, such as the license plate in the "Jin" word, can be perfectly extracted from the outline, but directly using the outline operation is not appropriate. This method of contour can be applied to English or numeric characters at the back of the license plate. Chinese must be dealt with separately. The character "E" after the word "Su" stands for the city code, and there is a symbol between it and the character after it, increasing the gap between the two characters. Find this character and offset it to the left and then the external matrix of the Chinese character can be found. Figure 3 is the "city code" and the " Provincial abbreviate" rectangle that has been deduced from it. For getting this "special character," as shown in Figure 3, the "special character" lies between one-seventh and two-seventh of the license plate. All character matrices can be successfully obtained as follows:

\section{BHIUIKIILI2}

Fig. 4 blocks that are intercepted and normalized.

\section{CHARACTERS RECOGNITION}

There are many methods for character recognition of license plate image, such as template matching method, pattern recognition algorithm, artificial neural network recognition algorithm, structural feature recognition algorithm and statistical feature recognition algorithm.

For character recognition based on simple template matching algorithm, we first need to set up character template. The A-Z, 0-9, and the Chinese characters representing the municipalities, provinces (or special military and police vehicles) which constitute the vehicle license plates are collected to form a character library.

The character image of vehicle license plate after character segmentation is matched one by one with the characters in the template library to recognize the characters. However, after character segmentation, the size of the character image is often different, so the character must be normalized.That is the vehicle license plate character image is scaled linearly to a $9 * 16$ dot matrix image, so that its size is consistent with the character of the standard module. Then the difference and similarity between the character image and the character template are calculated. Generally, the difference is less than $30 \%$, and the similarity is more than $95 \%$, the matching can be considered.Template-based matching method only needs to match some features of the target, so that the workload is less and the recognition speed is higher.

There are many algorithms for calculating template matching degree. Usually adopted a pattern as forllow:

$$
C_{f g}=\frac{100}{100} \sum_{i=1}^{M} \sum_{j=1}^{N} \frac{\left|f_{i j} \wedge g_{i j}\right|}{T_{f}+T_{g}}
$$

Among them, $\wedge$ is the arithmetic; $\mathrm{f}$ is the template binary image, , $\mathrm{G}$ is the corresponding license plate binary image; $T_{f}$ and $T_{g}$ are corresponding to the number of pixels in the binary image with the value of 1 ; the size of the two images is the same, both are $\mathrm{M} * \mathrm{~N}$.

\section{VI.CONCLUDING REMARKS}

Although the license plate recognition technology has been relatively mature in the static environment of $\mathrm{HD}$, the difficulties still exist in special scenes such as fog and haze, angle of incorrect view and darkness, especially in the future of unmanned traffic, unmanned vehicles, license plate recognition technology has a lot of room for improvement.

This paper discusses the four steps of license plate recognition, including image preprocessing, license plate location, character segmentation and character recognition. In the image pre-processing stage, the de-fogging, gray and binary processing are added, which greatly improves the image accuracy. Then the spatial domain filtering is used to get an accurate location image for the license plate. In the license plate positioning stage, the license plate position can be determined by edge detection, and the license plate can be accurately positioned by tilting correction. This paper analyzes and compares the edge detection methods, and solves the problems of insufficient image quality, batch and automatic recognition of vehicle license plate effectively by optimizing and improving Canny algorithm. In the phase of character segmentation and character recognition, a solution is proposed to segment Chinese characters by character contour method. The method of precise character segmentation and template matching are studied to realize automatic license plate recognition. Through the research and analysis of the digital image processing technology in the license plate recognition system, it lays a solid foundation for updating the future license plate automatic recognition system and improving the recognition ability.

\section{REFERENCES}

[1] Zhao Shan, et al. Research on License Plate Recognition Technology Based on Matlab Image Processing [J].Surveying and Mapping Engineering, 2014, 23 (1): 70-72

[2] Zhuo Junyu, Ho Zhihui. Research and Implementation of a License Plate Recognition and Location Method Based on Open CV [J].China Management Informatization, 2015,18(13): 209-210.

[3] OLIVA, TORRALBA. Modeling the shape of the scene: a holistic representation of the spatial envelope [J]. International Journal of Computer Vision, 2001, 42 (3): 145-175.

[4] Zhang Tao, Wang Jiankui, Zhang Guoshan, et al. Image preprocessing algorithm for license plate recognition system in foggy weather [J]. Journal of Shandong University of Technology: Natural Science Edition, 2015, 29 (1): 15-19.

[5] HE Kaiming, SUN Jian, TANG Xiaoou. Single image haze removal using dark channel prior [J]. IEEE Transactions on Pattern Analysis and Machine Intelligence, 2011, 33 (12): 2341-2353.

[6] HE Kaiming, SUN Jian, TANG Xiaoou. Guided image filtering [J]. IEEE Transactions on Pattern Analysis and Machine Intelligence, 2013 36 (6): 1397-1409

[7] Gonzales. Digital image processing Matlab edition [M]. Beijing: Publishing House of electronics industry, 2007.

[8] D.P. Mukherjee Debasish Jana. Computer Graphics Algorithms and Implementation [M]. Beijing: Tsinghua University Press, 2012.

[9] Xie Fengying. Digital image processing and application [M]. Beijing: Publishing House of electronics industry, 2014

[10] Li Junshan, Li Xuhui. Digital image processing [M]. Beijing: Tsinghua University press, 2007

[11] Zeng Quan, Tan Beihai. License Plate Recognition System Based on SVM and BP Neural Network [J]. Electronic Technology, 2016, 29 (1): 98-101. 
[12] Sun Jinling, et al. License Plate Image Location Based on Color Characteristics and Improved Canny Operator [J]. Journal of Jilin University (Science Edition), 2015, 53 (4): 693-697

[13] Jiang Zhihua, et al. An unconventional method for license plate tilt correction [J].Computer Applied Research, 2006,23(3): 175-177. 